\title{
Implementación y valoración de seminarios preparatorios para las prácticas externas en el Grado de Pedagogía.
}

\section{Implementation and assessment of the preparatory workshops for external practies in the degree of Pedagogy.}

\section{Dr. Juan Pablo Hernández Ramos}

Coordinador de prácticas Facultad de Educación, curso 2015-2016 Universidad de Salamanca juanpablo@usal.es

\section{Dra. Sonia Casillas Martín}

Coordinadora de prácticas Grado de Pedagogía , curso 2015-2016

Universidad de Salamanca scasillasma@usal.es

Hernández Ramos, J.P. \& Casillas Martín, S. (2017). Implementación y valoración de seminarios preparatorios para las prácticas externas en el Grado de Pedagogía. Revista Prácticum, 2(1), 40-59. 


\section{Resumen}

Las prácticas externas se han convertido en los últimos años en un importante componente de la formación universitaria, destacando este período no sólo como un momento de acercamiento al mundo laboral, sino como el período idóneo para englobar y aplicar todas las competencias desarrolladas previamente. Asociado a un proyecto de innovación y mejora de la calidad docente, mediante una metodología no experimental, este estudio pretende valorar y analizar la importancia de los seminarios preparatorios de las prácticas externas para los estudiantes de $4^{\circ}$ de Pedagogía. Los resultados nos muestran como los estudiantes valoran positivamente la realización de dichas actividades formativas, no sólo por ofrecerles un nexo con el mercado laboral, sino porque les ayuda a elegir el lugar donde realizar sus prácticas externas. Iniciativas como la reflejada en este artículo demuestran la preocupación de las universidades por garantizar la calidad de la enseñanza universitaria, tanto dentro, como fuera de sus facultades.

\section{Abstract}

External practices have become in recent years an important component of university education, highlighting this period not only as a moment of approach to the world of work, But as the ideal period to encompass and apply all previously developed competencies. Associated with a project of innovation and improvement of teaching quality, through a non-experimental methodology, this study aims to evaluate and analyze the importance of the seminars preparatory to the external practices of students of fourth degree in Pedagogy. The results show us how students positively value the performance of these training activities, not only by offering them a link with the labor market, but also because it helps them to choose the place where to carry out their external practices. Initiatives as reflected in this article demonstrate the concern of universities to ensure the quality of university education, both inside and outside their faculties.

\section{Palabras clave}

Prácticum, Prácticas externas, universidad, Aprendizaje experiencial.

\section{Keywords}

Practicum, External Practices, University, Experiential learning. 


\section{Introducción}

Desde el momento en que se diseñaron y aprobaron los diferentes grados bajo los criterios de convergencia europea estipulados por el Plan Bolonia para las universidades europeas, desde la Facultad de Educación de la Universidad de Salamanca (USAL) se están desarrollando constantes investigaciones y avances en pos de la mejora educativa; destacando la progresiva importancia que está adquiriendo el Practicum en los Planes de estudio de las diferentes titulaciones (Zabalza, 2011). El Espacio Europeo de Educación Superior (EEES) instauró un nuevo escenario para el acercamiento de la universidad al mundo laboral al ponerle mayor énfasis a prácticas externas (Valverde, 2016).

Existe una realidad social en la que, debido al avance tecnológico, el mundo está interconectado y todo se puede localizar, exponer, intercambiar, transferir, recibir, vender o comprar sin importar el lugar en que nos encontremos (De Pablos, 2010); por ello, es necesario que todas las instituciones de educación superior mejoren y se adapten a los cambios, para así poder afrontar las nuevas demandas educativas de la sociedad de la información. La universidad, y particularmente los profesores universitarios, tienen la responsabilidad de contribuir, con una práctica educativa innovadora, en la formación integral de individuos con competencias suficientes para incorporarse y desenvolverse fácilmente en la realidad que tengan que vivir (González, 2008).

Este artículo pretende reflejar el proceso de diseño, implementación y valoración de los seminarios preparatorios para las prácticas externas del Grado de Pedagogía en el curso 2015-2016; iniciativa desarrollada desde la coordinación de prácticas de la titulación, con la finalidad de optimizar el grado de adquisición y desarrollo de competencias por parte de los estudiantes de dicha titulación durante la preparación y el desarrollo de las citadas prácticas.

El Practicum, dentro de las titulaciones de grado, resulta un importante componente de la formación universitaria que se ofrece a los estudiantes (Alemany \& Perramon, 2011; Mendoza \& Covarrubia, 2014), periodo que en ocasiones es descuidado o planificado de manera superficial; considerando, por tanto, que cualquier tipo de perfeccionamiento en la planificación y desarrollo de las prácticas externas es una mejora en la oferta académica de las universidades. Las prácticas regladas contribuyen al logro efectivo de toda una serie de conocimientos, competencias y actitudes profesionales que precisarán los titulados en Pedagogía para el desempeño de su labor profesional (Gavari, 2012; González \& Hevia, 2011). Por todo ello, se considera que el Practicum debe diseñarse, implementarse y 
evaluarse, otorgándole la importancia debida, al entenderlo como eje vertebrador y globalizador de la titulación (Zabalza, 2011).

El sistema universitario español tampoco ha estado ajeno al valor formativo del prácticum en la preparación integral de sus estudiantes (Mendoza \& Covarrubias, 2014). La importancia otorgada al practicum por el proceso de convergencia europea queda reflejada en la aparición de infinidad de estudios en España centrados en la valoración y mejora del periodo de prácticas de los estudiantes (Alemany \& Perramon, 2011; Bartolome, Cantón, \& Moral, 2016; Valverde, 2016). Destacando incluso la existencia de investigaciones previas centradas en los grados de magisterio (González-Sanmamed \& Fuentes-Abeledo, 2011; Mendoza \& Covarrubias, 2014) e incluso en el propio grado de pedagogía (González \& Hevia, 2011; Hevia, 2010).

La implantación del EEES, en lo referente al Practicum, ha conllevado que las universidades, se preocupen por diseñar e implementar mecanismos que permitan garantizar unas prácticas de calidad para nuestros estudiantes; o como mínimo al mismo nivel que el resto de actividades formativas (Alemany \& Perramon, 2011).

\section{Estado de la cuestión}

Desde siempre, se entiende el periodo de prácticas de las titulaciones universitarias como un tiempo en que el estudiante abandona la facultad para observar y colaborar en una función laboral similar a para la que se está preparando. En base al Real Decreto $592 / 2014$, de 11 de julio, por el que se regulan las prácticas académicas externas; éstas constituyen una actividad de naturaleza formativa realizada por los estudiantes y supervisada por las universidades, cuyo objetivo es permitir a los mismos aplicar y complementar los conocimientos adquiridos en su formación académica, favoreciendo la adquisición de competencias que les preparen para el ejercicio de actividades profesionales, faciliten su empleabilidad y fomenten su capacidad de emprendimiento. No obstante, esta labor formativa, viene reforzada por la necesidad, reflejada en los fundamentos del EEES, de acercar al estudiante al mundo laboral. Cuando se habla de Practicum estamos integrando en esa categoría todo un conjunto de actuaciones curriculares con contenido y sentido diferente al dado tradicionalmente (Zabalza, 2016).

En la fundamentación inicial de este Real Decreto 592/2014 se hace mención de las exigencias del proceso de construcción del 


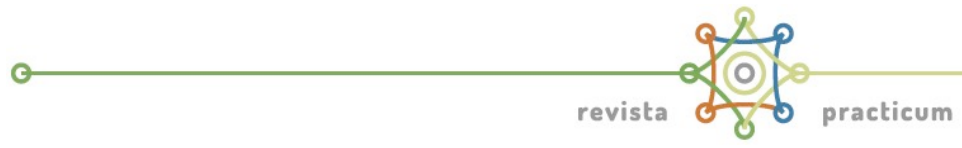

Espacio Europeo de Educación Superior al mencionar en diversas leyes manifiesta que:

"... se ha puesto un especial énfasis en la realización de prácticas externas por los estudiantes universitarios, previendo que los planes de estudios de Grado contendrán «toda la formación teórica y práctica que el estudiante deba adquirir», entre la que se mencionan «las prácticas externas» (artículo 12.2), y que «si se programan prácticas externas, estas tendrán una extensión máxima de 60 créditos y deberán ofrecerse preferentemente en la segunda mitad del plan de estudios» (artículo 12.6)".

Aunque pueda parecer que se intenta justificar las prácticas por su importancia como acercamiento al mundo laboral (Mendoza \& Covarrubias, 2014), no se debe descuidar la finalidad de completar los aprendizajes y la formación que se obtiene en la universidad, ya que al final lo trascendental es que los estudiantes aprenden durante el proceso (Zabalza, 2016). Autores como De Miguel (2006), a la hora de suponer dichos propósitos educativos orientados en el desarrollo de competencias dentro del Plan Bolonia, destacan siete modalidades de enseñanza entre la que encontramos las prácticas externas. Dicho autor considera las prácticas externas como "el conjunto de actuaciones que un estudiante realiza en un contexto natural relacionado con el ejercicio de una profesión" (2006, p.64). Para poder delimitar mejor esta modalidad docente, en base a otros estudios (Hernández, 2014) y teniendo siempre presente la obra de De Miguel (2006) se elabora la tabla 1.

\begin{tabular}{l|l}
\hline Finalidad & $\begin{array}{l}\text { Completar la formación de los estudiantes en el propio } \\
\text { lugar en el que tendrán que desenvolverse en el } \\
\text { mundo laboral. }\end{array}$ \\
\hline Escenario & Contextos profesionales vinculados a la titulación. \\
\hline Ventajas & $\begin{array}{l}\text { Acercamiento al mundo laboral y a la realidad social. } \\
\text { Desarrollo de actitudes y valores ligados al desempeño } \\
\text { de una profesión. }\end{array}$ \\
\hline \multirow{2}{*}{ es } & $\begin{array}{l}\text { Sitúan al estudiante en un marco reducido y centrado } \\
\text { en una profesión. } \\
\text { Enfrentan al estudiante a una acción muy diferente de } \\
\text { la que realizan en las universidades. } \\
\text { Dependiendo del lugar de acogida, el grado de } \\
\text { actuación del estudiante puede ser muy diferente. }\end{array}$ \\
\hline
\end{tabular}




\begin{tabular}{l|l}
\hline $\begin{array}{l}\text { Descripción } \\
\text { básica }\end{array}$ & $\begin{array}{l}\text { El estudiante abandona la facultad para ser acogido } \\
\text { temporalmente en el mundo laboral. }\end{array}$ \\
\hline & Tabla 1. Prácticas Externas (Adaptado de De Miguel) (2006)
\end{tabular}

\subsection{Seminarios previos a las prácticas de Pedagogía}

En una sociedad dinámica como la actual, el rol del pedagogo ha adquirido infinidad de posibilidades laborales. Mediante la realización de los seminarios preparatorios, el estudiante de pedagogía podrá acercarse a las diferentes posibilidades y tendrá mayor información a la hora seleccionar el lugar en que desea realizar su periodo de prácticas. Con ello, pretendemos garantizar las dos cuestiones que los diferentes estudios previos destacan como relevantes para el aprovechamiento de las prácticas externas: que las experiencias vividas sean significativas para la futura profesión de los estudiantes (González \& Hevia, 2011; Zabalza, 2016); y que el contexto en el que se desarrollen fomente un aprendizaje novedoso para ellos, tanto en el momento de realización, como posteriormente cuando ejerzan como pedagogos (Alemany \& Perramon, 2011; Bartolome et al., 2016; Zabalza, 2016).

Teniendo en cuenta que lo normal es que cada alumno sólo conozca la realidad laboral de una de las múltiples funciones del pedagogo, los seminarios prácticos, no dejan de tener una función formativa de acercamiento entre la universidad y el mercado laboral. Los seminarios previos a la realización de las prácticas adquieren diferentes finalidades según el artículo 3 del Real Decreto 592/2014 recoge textualmente que:

"a. Contribuir a la formación integral de los estudiantes complementando su aprendizaje teórico y práctico.

b. Facilitar el conocimiento de la metodología de trabajo adecuada a la realidad profesional en que los estudiantes habrán de operar, contrastando y aplicando los conocimientos adquiridos.

c. Favorecer el desarrollo de competencias técnicas, metodológicas, personales y participativas.

d. Obtener una experiencia práctica que facilite la inserción en el mercado de trabajo y mejore su empleabilidad futura.

e. Favorecer los valores de la innovación, la creatividad y el emprendimiento".

Generalmente, los estudiantes de Pedagogía consiguen un alto grado de acercamiento a una de las muchas realidades laborales 


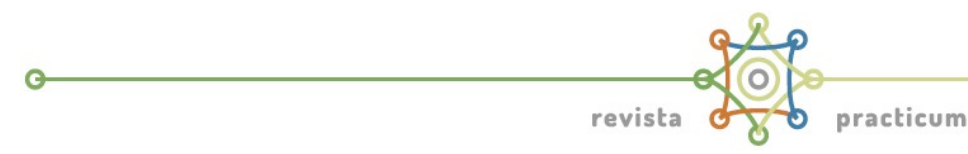

existentes como graduados en Pedagogía. Mediante los seminarios previos a las prácticas externas, no sólo se pretende que el estudiante conozca todos los ámbitos de intervención profesional relacionados con el grado; sino que también tenga un acercamiento a los centros, programas e instituciones que tienen algún tipo de convenio con la Universidad de Salamanca- Algunas empresas o instituciones son muy enriquecedoras para los estudiantes y en ellas tendrán oportunidad de experimentar actividades prácticas vinculadas con su profesión, no obstante, en una titulación que no tiene las salidas profesionales totalmente marcadas, es importante poder realizar un acercamiento a otras posibles actividades laborales. Además, al entender el Practicum como momento y situación de aprendizaje, importa mucho tomar en consideración de qué manera se ha organizado ese proceso (Zabalza, 2016); y dedicarle el tiempo necesario a la planificación, organización y asignación de los estudiantes.

Los seminarios preparatorios consisten en breves sesiones informativas, de aproximadamente 45 minutos de duración, en donde los diferentes responsables de los centros e instituciones colaboradoras con la universidad acercan a los estudiantes a su realidad laboral diaria. Posteriormente, tras la realización de los diferentes seminarios, durante la sesión final, se realiza la asignación del centro, institución o programa dónde el alumno realizará las prácticas, de acuerdo con su solicitud y con los criterios establecidos por la comisión de prácticas, así como la asignación al alumno de un profesor tutor (profesor/a de la universidad) y de un supervisor (de la institución asignada). La asignación de diferentes profesores de la facultad como tutores, involucra a un gran número de profesores con diferentes opiniones, pudiendo generar cierto grado de confusión en los estudiantes (Bartolome et al., 2016); hecho que mediante la realización de los seminarios preparatorios de manera conjunta se pretende prevenir.

\section{Metodología}

\subsection{Diseño}

La metodología de investigación seleccionada ha sido no experimental transversal, del tipo ex post facto, en donde no se modifica ni manipula ninguna de las variables y simplemente se recogen e interpretan las medidas de manera válida y fiable (Pérez, Galán, \& Quintanal, 1992; Kerlinger \& Lee, 2002) mediante análisis descriptivos. Así mismo, teniendo en consideración que, independientemente de cuál sea la estructura y el propósito formativos del Practicum, éste tiene siempre una dimensión personal 
que desborda ampliamente los objetivos planificados (Zabalza, 2016); al igual que en investigaciones previas (González \& Hevia, 2011; Mendoza \& Covarrubias, 2014), se considera necesario centrarse en los estudiantes, concretamente en su opinión posterior a la realización del Practicum.

\subsection{Finalidad y Objetivos}

La investigación recogida en este artículo, nace desde la figura del Coordinador de Prácticas de la Facultad de Educación de la Universidad de Salamanca, el cual, en concordancia con la Coordinadora de Prácticas de la Titulación de Pedagogía y la decana de la Facultad de Educación, consideran necesario optimizar el grado de adquisición y desarrollo de competencias por parte de los estudiantes de Pedagogía durante el desarrollo de las Prácticas Externas y la realización de los seminarios preparatorios para éstas.

Por tanto, el objetivo principal establecido consiste en analizar el desarrollo de las prácticas externas de los estudiantes de Pedagogía; así como la realización de seminarios preparatorios. Objetivo concordante con la importancia otorgada por ANECA, durante los procesos de evaluación de las titulaciones universitarias, a las prácticas curriculares.

De cara a facilitar la consecución del objetivo principal del proyecto se establecen tres objetivos más específicos: valorar los factores influyentes en el desarrollo de las prácticas externas; facilitar a los estudiantes la elección del lugar de realización de sus prácticas; y mostrar al futuro pedagogo un nexo entre la universidad y el mundo laboral.

\subsection{Población y muestra}

La población de estudio considerada se compone por los 60 estudiantes del Grado de Pedagogía matriculados en la asignatura obligatoria Practicas Externas el curso 2016-2017. Tras la aplicación del cuestionario de manera presencial, se obtienen 43 respuestas, quedando establecida la muestra en dicha cantidad.

\subsection{Temporalización y fases}

Como queda reflejado en la tabla 2 , la investigación comienza en las semanas previas al inicio del 2015-2016 y continúa hasta el curso 2016-2017, momento en que se procede tanto a la difusión de los resultados, como a la implementación de las decisiones tomadas a partir de los resultados obtenidos. Se pueden diferenciar cinco grandes fases: preparación, desarrollo, realización, evaluación y difusión. 
Las primeras actuaciones para la consecución de los seminarios preparatorios se dieron la primera semana del mes de septiembre de 2015, cuando, una vez conocido el calendario disponible, se seleccionan los profesionales y se empiezan a establecer los primeros contactos con los diferentes profesionales. Después de varios acercamientos, al final, debido a las limitaciones de horario que teníamos al disponer solo la mañana el viernes, tras contactar con cerca de una veintena de profesionales, se consiguió acordar la implicación de once profesionales de los diferentes ámbitos profesionales.

\begin{tabular}{lllll}
\hline & FASE & DESCRIPCIÓN & TEMPORALIZ \\
ACIÓN
\end{tabular}




\begin{tabular}{|c|c|c|c|}
\hline & $\begin{array}{l}\text { de recogida de } \\
\text { información. }\end{array}$ & $\begin{array}{l}\text { realización de las prácticas y la adquisición } \\
\text { y desarrollo de competencias durante } \\
\text { éstas. }\end{array}$ & \\
\hline & $\begin{array}{l}\text { Aplicación del } \\
\text { instrumento. }\end{array}$ & $\begin{array}{l}\text { Aplicación a los } \text { estudiantes del } \\
\text { instrumento diseñado y validado para ello. }\end{array}$ & Marzo 2016 \\
\hline & $\begin{array}{l}\text { Análisis } \\
\text { datos. }\end{array}$ & Análisis de los datos. & $\begin{array}{l}\text { Abril, Mayo y } \\
\text { Junio de } 2016\end{array}$ \\
\hline 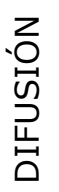 & $\begin{array}{l}\text { Difusión de } \\
\text { resultados. }\end{array}$ & $\begin{array}{l}\text { Difusión de resultados mediante artículos y } \\
\text { participación en eventos. }\end{array}$ & $\begin{array}{l}\text { Curso } \\
2017\end{array}$ \\
\hline
\end{tabular}

Tabla 2. Calendario de realización (Elaboración Propia)

Durante los meses de octubre y noviembre de 2015, contamos con los siguientes profesionales y entidades:

- Una fundación de carácter cultural y educativo, sin ánimo de lucro, creada por el Ayuntamiento de Salamanca.

- Representantes de varios programas de la propia universidad: Servicio de Asuntos Sociales y Programa Interuniversitario de la Universidad de la Experiencia.

- Diferentes asociaciones: asociación de padres, familiares y amigos de niños oncológicos de Castilla y León; y asociación salmantina de equinoterapia.

- Una empresa privada de acción pedagógica.

- Una orientadora de un centro público.

- Representantes de dos residencias juveniles.

- Programa de apoyo al estudio para miembros de un equipo de futbol.

- Miembros del Servicio de Inspección de Salamanca.

Tras la realización de los diferentes seminarios preparatorios se les concede a los estudiantes un periodo de reflexión y posteriormente se realiza la asignación de los estudiantes en las diferentes posibilidades seleccionadas. En caso de existir varias personas interesadas en un mismo centro u organización, se decide recurrir a un sorteo público. De las plazas ofertadas, en sólo tres de ellas se tuvo que recurrir a dicho sorteo, quedando patente la gran variedad de intereses de los 60 estudiantes matriculados.

Una vez terminado el periodo de prácticas, durante una de las clases posteriores se aplica en papel un cuestionario de valoración de los seminarios preparatorios. Tras la posterior recogida y análisis de 


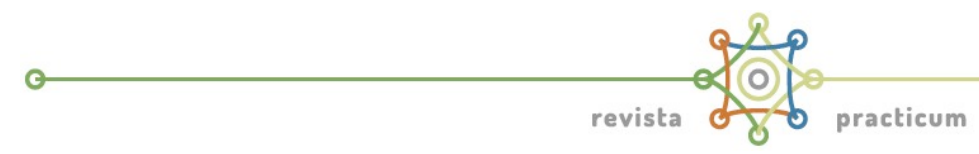

datos, se procede a la elaboración del informe de mejora del proceso y a la difusión de los resultados, siendo este articulo la comunicación principal.

\subsection{Instrumento}

Como se acaba de anticipar, de cara a la recogida válida y fiable de información, se emplea como instrumento un cuestionario diseñado para la propia ocasión. El cuestionario es una técnica de recogida de información que supone un interrogatorio en que las preguntas establecidas de antemano, se plantean siempre en el mismo orden y se formulan con los mismos términos, empleándose está práctica comúnmente en enfoques y diseños de investigación típicamente cuantitativos (Rodríguez, Gil, \& García, 1996) como el que acontece.

Previamente a la elaboración del cuestionario, se realizó un proceso de reflexión sobre el tipo de información que se deseaba obtener $y$, por consiguiente, sobre el tipo de preguntas y el modo de plantearlas (Hernández, Martínez, García, Herrera, \& Rodríguez, 2013). Y es que, como destacan ciertos autores "la planificación de un cuestionario implica diseñar un conjunto de cuestiones que supongan concretar las ideas, creencias o supuestos del encuestador en relación con el problema de estudio" (Rodríguez et al., 1996, p. 187). Tras dicho proceso, se confecciona un cuestionario en donde se diferencian tres apartados:

- El apartado inicial está formado por 7 proposiciones donde mediante una tipo Likert (Morales, Urosa, \& Blanco, 2003) con cinco posibilidades de respuesta: totalmente en desacuerdo (1); en desacuerdo (2); ni acuerdo, ni desacuerdo (3); de acuerdo (4) y totalmente de acuerdo (5); el estudiante manifiestan su opinión sobre los seminarios y su utilidad.

- Posteriormente, el estudiante valora los 11 seminarios de manera independiente; indicando de cada uno de ellos, la opinión sobre la duración (corto, adecuado o largo) y la evaluación del seminario en sí (escala de 1 a 5). Para dicha valoración, los estudiantes deben considerar el interés, la utilidad y la relevancia de los aspectos comentados en el seminario en cuestión.

- El cuestionario concluye con un apartado final, con dos preguntas de valoración global, con dos únicas posibilidades de respuesta: $\mathrm{Si}$ y NO. Estás preguntas, solamente las responderán los estudiantes que no se habían llegado a un acuerdo previo con alguna institución para realizar las prácticas. 
Posteriormente, para valorar la fiabilidad o consistencia interna del cuestionario se procedió al cálculo del coeficiente de a de Cronbach, obteniendo un valor de .834. En base a este indicador, al ser una puntuación superior a .7, se considera un instrumento válido (Morales et al., 2003). Esto quiere decir que los ítems propuestos son precisos en su medición y valoran de manera consciente las opiniones de los estudiantes de Pedagogía sobre los seminarios preparatorios.

Aunque se considera la posibilidad de realizar el cuestionario bajo un diseño electrónico, para conseguir que sea cumplimentado por el mayor número de estudiantes posibles, se planifica un diseño en formato tradicional de papel y una aplicación anónima en el aula antes del desarrollo de una asignatura obligatoria de la titulación.

\section{Análisis de resultados}

Aprovechando la existencia de tres grandes apartados, a continuación, se exponen los resultados en base a dicha estructuración:

\subsection{Valoración de los seminarios}

En base a los resultados expuestos en la taba 4, se puede considerar que los estudiantes de cuarto de Pedagogía consideran que los seminarios preparatorios son útiles, relevantes e interesantes. Destacando el convencimiento manifestado en los ítems 2: Gracias a los seminarios he conocido diferentes salidas laborales; y 3: Los seminarios me han ofrecido un nexo entre la universidad y el mercado laboral, destacando la necesidad de establecer actividades que conecten la formación con el mercado laboral.

Sin embargo, a la hora de analizar los resultados obtenidos en otros ítems, vemos que, aunque los seminarios han servido para motivar a los estudiantes para acudir a las prácticas (ítem 4: Los seminarios han hecho que acuda a las prácticas con una motivación mayor), no han servido para aumentar la vocación pedagógica de los alumnos (ítem 5: La asistencia a los seminarios ha aumentado mi vocación hacia la Pedagogía) y tampoco, en parte debido a la estructura de realización tan compacta, han ofrecido lugares de debate profesional (ítem 6: Los seminarios me han ofrecido un lugar donde debatir sobre el rol del pedagogo).

Así mismo, si observamos los resultados obtenidos en el ítem 7: Los seminarios me parecen necesarios para el buen funcionamiento de las prácticas; y en el ítem 1: Los seminarios me han ayudado a 


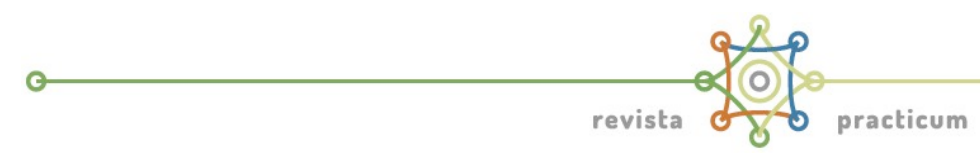

decidir el lugar donde realizar las prácticas; se entiende que los seminarios preparatorios son considerados como útiles.

\begin{tabular}{|c|c|c|c|c|c|c|c|c|}
\hline & Media & $\begin{array}{l}\text { Desv. } \\
\text { Tip. }\end{array}$ & $\begin{array}{l}1 \\
(\%)\end{array}$ & $\begin{array}{l}2 \\
(\%)\end{array}$ & $\begin{array}{l}3 \\
(\%)\end{array}$ & $\begin{array}{l}4 \\
(\%)\end{array}$ & $\begin{array}{l}5 \\
(\%)\end{array}$ & $\mathrm{N}$ \\
\hline $\begin{array}{l}01 \text {. Los seminarios me han } \\
\text { ayudado a decidir el lugar donde } \\
\text { realizar las prácticas. }\end{array}$ & 3,37 & ,926 & 2,3 & 16,3 & 30,2 & 44,2 & 7 & 43 \\
\hline $\begin{array}{l}\text { 02. Gracias a los seminarios he } \\
\text { conocido diferentes salidas } \\
\text { laborales. }\end{array}$ & 3,91 & ,684 & 0 & 0 & 27,9 & 53,5 & 18,8 & 43 \\
\hline $\begin{array}{l}\text { 03. Los seminarios me han ofrecido } \\
\text { un nexo entre la universidad y el } \\
\text { mercado laboral. }\end{array}$ & 3,44 & 700 & 0 & 7 & 46,5 & 41,9 & 4,7 & 43 \\
\hline $\begin{array}{l}\text { 04. Los seminarios han hecho que } \\
\text { acuda a las prácticas con una } \\
\text { motivación mayor. }\end{array}$ & 3,38 & 987 & 2,4 & 16,7 & 33,3 & 35,7 & 11,9 & 42 \\
\hline $\begin{array}{l}\text { 05. La asistencia a los seminarios } \\
\text { ha aumentado mi vocación hacia la } \\
\text { Pedagogía. }\end{array}$ & 3,33 & ,865 & 2,3 & 11,6 & 44,2 & 34,9 & 7 & 43 \\
\hline $\begin{array}{l}\text { 06. Los seminarios me han ofrecido } \\
\text { un lugar donde debatir sobre el rol } \\
\text { del pedagogo. }\end{array}$ & 3,21 & ,940 & 2,3 & 18,6 & 44,2 & 25,6 & 9,3 & 43 \\
\hline $\begin{array}{l}\text { 07. Los seminarios me parecen } \\
\text { necesarios para el buen } \\
\text { funcionamiento de las prácticas. }\end{array}$ & 3,52 & 1,110 & 2,4 & 19 & 23,8 & 33,3 & 21,4 & 42 \\
\hline
\end{tabular}

Tabla 4. Valoración de los seminarios (Elaboración propia)

\subsection{Valoración específica de cada seminario}

Como se ha comentado con anterioridad, se considera necesario incorporar un apartado en que se valoren específicamente los 11 diferentes seminarios, indicando de cada uno de ellos, la opinión sobre la duración mediante tres opciones de respuesta y la valoración del seminario teniendo en cuenta el interés, la utilidad y la relevancia de los aspectos comentados durante la realización de cada seminario.

Los resultados obtenidos se pueden observar en la tabla 5 , en donde de manera general, los estudiantes consideran adecuada la duración de los seminarios, la cual ha sido de manera general de unos 50 minutos. En lo que se refiere a los seminarios, los menos valorados han sido el primero y el décimo, con puntuaciones que no llegan al 3. Por ello, remarcar la relación de esas valoraciones menos positivas con el aumento de opiniones que consideraban dichos seminarios como largos. 
Por lo demás, de manera general, la valoración media obtenida por el grueso de los seminarios, omitiendo los dos casos citados con anterioridad, en una escala que oscila desde 1 hasta 5 , ha fluctuado entre 3,14 y 3,92 .

\begin{tabular}{|c|c|c|c|c|c|c|}
\hline & \multicolumn{2}{|c|}{ Valoración } & \multicolumn{4}{|c|}{ Duración } \\
\hline & Media & $\begin{array}{l}\text { Desv. } \\
\text { Tip. }\end{array}$ & $\begin{array}{l}\text { Corto } \\
\%\end{array}$ & $\begin{array}{l}\text { Adecuado } \\
\%\end{array}$ & $\begin{array}{l}\text { Largo } \\
\%\end{array}$ & $\mathrm{~N}$ \\
\hline $\begin{array}{l}\text { 1. Programa cultural del } \\
\text { Ayuntamiento }\end{array}$ & 2,85 & 1,027 & 2,50 & 62,50 & 35,00 & 40 \\
\hline $\begin{array}{l}\text { 2. Servicio asuntos Sociales } \\
\text { USAL }\end{array}$ & 3,44 & 821 & 2,56 & 79,49 & 17,95 & 39 \\
\hline $\begin{array}{l}\text { 3. Programa Interuniversitario } \\
\text { de la Universidad de la } \\
\text { Experiencia }\end{array}$ & 3,48 & 1,062 & 10,00 & 82,50 & 7,50 & 40 \\
\hline $\begin{array}{l}\text { 4. Asociación de Padres, } \\
\text { Familiares y amigos e niños } \\
\text { oncológicos de Castilla y León }\end{array}$ & 3,67 & ,772 & 2,56 & 92,31 & 5,13 & 39 \\
\hline $\begin{array}{l}\text { 5. Empresa privada: ámbito de } \\
\text { la Psicopedagogía }\end{array}$ & 3,30 & 1,381 & 2,50 & 85,00 & 12,50 & 40 \\
\hline 6. Residencia Juvenil I & 3,81 & 1,126 & 5,41 & 86,49 & 8,11 & 37 \\
\hline $\begin{array}{l}\text { 7. Orientadora en centro } \\
\text { publico }\end{array}$ & 3,14 & 1,134 & 5,41 & 75,68 & 18,92 & 37 \\
\hline $\begin{array}{l}\text { 8. Residencia Juvenil de } \\
\text { reinserción social }\end{array}$ & 3,92 & ,906 & 11,11 & 75,00 & 13,89 & 36 \\
\hline 9. Club de Futbol & 3,53 & 1,109 & 50,00 & 50,00 & 0,00 & 38 \\
\hline $\begin{array}{l}\text { 10. Servició de Inspección de } \\
\text { Salamanca }\end{array}$ & 2,74 & 1,107 & 0,00 & 57,89 & 42,11 & 38 \\
\hline $\begin{array}{l}\text { 11. Asociación Salmantina de } \\
\text { Equinoterapia }\end{array}$ & 3,58 & 1,030 & 0,00 & 100,00 & 0,00 & 38 \\
\hline
\end{tabular}

Tabla 5. Valoración individual de cada seminario (Elaboración propia)

\subsection{Valoración global}

Para concluir, las últimas cuestiones se refieren a valorar aspectos más globales y del tipo organizativo, cuestiones que desde la coordinación necesitamos conocer para valorar el proceso de manera global. 


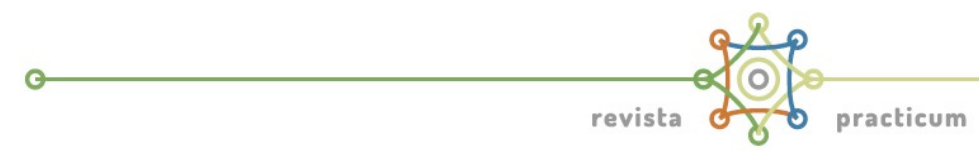

La primera de las consideraciones es clara y directa: ¿Los seminarios te han ayudado a elegir el lugar de realización de las prácticas?; y como se observa en la figura 1, de los 43 estudiantes, a 23 de ellos (53\%) los seminarios les han ayudado a decidir su lugar de prácticas. Este dato por sí sólo no se debe emplear para valorar los seminarios, ya que, en muchas ocasiones, como se muestra a continuación, los estudiantes tenían seleccionado su lugar de prácticas antes de la realización de los propios seminarios.

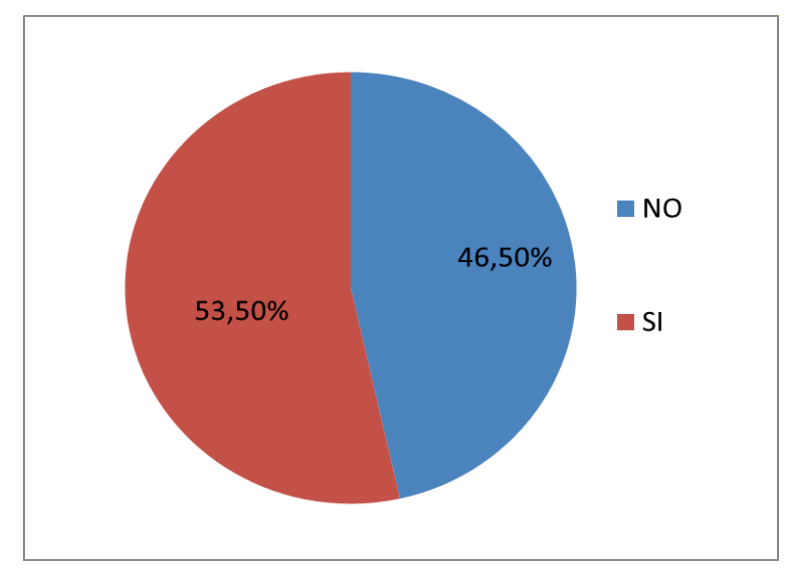

Gráfica 1. Pregunta general 1 (Elaboración propia)

Por ello, debemos centrarnos en las siguientes cuestiones y valorar conjuntamente los resultados; siendo vital conocer si ¿Los seminarios han hecho que cambien tus intenciones sobre el lugar de realización de las prácticas? Donde 15 de los 43 estudiantes $(34,9 \%)$ manifiestan que han cambiado sus intenciones, frente a $28(65,1 \%)$ que no se han visto influenciados. Teniendo en cuenta estos datos es importante tener en cuenta que 11 estudiantes se han buscado el centro de prácticas ellos previamente en base a otros intereses ajenos a la realización de los seminarios: intereses vocacionales, geográficos, laborales, etc. Por ello, en la tabla 6, al sólo responder

Si tenemos en cuenta solamente a los 32 estudiantes que al comenzar los seminarios no tienen decidido donde realizar las prácticas, la situación es bien distinta, ya que como se puede observar en la tabla 6 , al $62,5 \%$ de los estudiantes que no tenían preparadas y acordadas previamente sus prácticas, les ha servido para elegir su lugar de realización. Es más, incluso 12 estudiantes, el $37,5 \%$, ha cambiado de opinión y debido a la implementación de los seminarios ha modificado sus intenciones a la hora de elegir entidad para realizar las prácticas. 


\begin{tabular}{|c|c|c|c|c|c|}
\hline & SI & & NO & & $\mathrm{N}$ \\
\hline $\begin{array}{l}\text { ¿Los seminarios te han ayudado a elegir el } \\
\text { lugar de realización de las prácticas? }\end{array}$ & 20 & $62,5 \%$ & 12 & $37,5 \%$ & 32 \\
\hline $\begin{array}{l}\text { ¿Los seminarios han hecho que cambien } \\
\text { tus intenciones sobre el lugar de } \\
\text { realización de las prácticas? }\end{array}$ & 12 & $37,5 \%$ & 20 & $62,5 \%$ & 32 \\
\hline
\end{tabular}

Tabla 6. Valoración de los seminarios como ayuda para decidir el lugar de realización de las prácticas. (Elaboración propia)

\section{Discusión y Conclusiones}

La inclusión en los planes docentes de prácticas externas, con sus convenientes seminarios preparatorios, supone reconocer que, "además del conocimiento técnico y científico, es indispensable un conocimiento práctico que permita una aproximación a la realidad en la que se desenvolverán los futuros profesionales, desde el principio de aprender haciendo" (Mendoza \& Covarrubias, 2014, p. 113).

Así mismo, evaluar la calidad de la enseñanza universitaria se ha convertido en un tema necesario y actual. Conocer sus metas y sus objetivos y evaluar en qué grado se han alcanzado es un reto para las instituciones universitarias que desean lograr prestigio y competitividad (Alemany \& Perramon, 2011). En este momento, con el EEES implantado en la Universidad de Salamanca de manera general y en la Facultad de Educación de manera concreta, el estudiante debe hacer explícita su condición de miembro activo y ser considerado durante todo proceso evaluativo de su formación. Dentro de este nuevo paradigma asistido por el cambio en la conceptualización de la formación universitaria, el Practicum adquiere una nueva relevancia. Por ello se trata de intentar establecer nuevos criterios que garanticen la calidad del Practicum (Alemany \& Perramon, 2011; Zabalza, 2011). Dicha calidad, debe venir determinada por una buena planificación, un adecuado proceso de selección de los organismos colaboradores y una valoración conjunta de todo el proceso; teniendo significativa importancia el diseño, implementación y evaluación de los seminarios preparatorios.

El Plan de Mejoras de las Prácticas Externas de las diferentes titulaciones de la Facultad de Educación se rige por una dinámica de evaluación sistemática y participativa que permita un conocimiento riguroso de la institución como base para la toma de decisiones que garanticen la mejora continuada de la oferta educativa de la Facultad de Educación de la Universidad de Salamanca. En todo momento se debe considerar la importancia del practicum para la puesta en un 


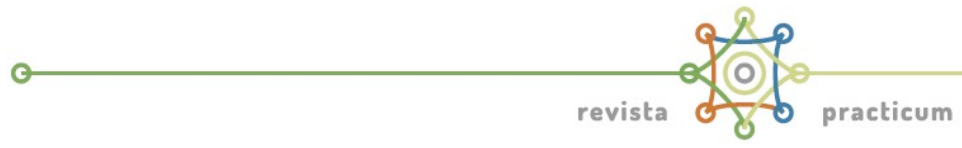

contexto real de las competencias desarrolladas durante las titulaciones, no obstante, como refleja Zabalza (2016, p. 4): "tiene el pecado original de que habiendo nacido muy vinculado a la práctica, al hacer, se habla más de experiencias que del fundamento y sentido curricular sobre el que dichas experiencias se basan".

Mediante la realización de esta investigación, se han valorado los seminarios preparatorios a la realización de las prácticas del grado de Pedagogía en la Universidad de Salamanca, con la intención de fomentar la motivación de los estudiantes durante este periodo, así como facilitar la elección de la entidad colaboradora en la que realizar dicho periodo. Estas actividades formativas de carácter preparativo sirven para conocer y considerar las circunstancias que interfieren en el desarrollo de competencias por parte de los estudiantes. Por ello, desde la coordinación de prácticas se podrán considerar y controlar de forma que se mejore el grado de aprovechamiento de las prácticas por parte del estudiante.

Así mismo, teniendo en cuenta que el profesional de la Pedagogía dispone de un amplio abanico de salidas profesionales: orientación, recursos humanos, gestión, evaluación, etc., con el desarrollo de los seminarios preparatorios se pretende acercar al estudiante a diversas salidas laborales, y no sólo a la relativa al lugar de realización de sus prácticas.

En lo referente a la toma de decisiones de cara al próximo curso, teniendo en cuenta los resultados expuestos y la importancia otorgada por los estudiantes se toman las siguientes decisiones:

- Mantener la realización de los seminarios preparatorios.

- Valorar la supresión o modificación de los dos seminarios valorados de manera más negativa por los estudiantes.

- Valorar la posibilidad de que no todos los seminarios tengan la misma duración, ya que se considera que las posibilidades expositivas de los profesionales son diferentes en función de sus actividades.

- Mantener el sistema de asignación de centros.

Desde una perspectiva global, la consolidación del EEES ha resaltado la importancia formativa del periodo de prácticas (Alemany \& Perramon, 2011; Bartolome et al., 2016; Gavari, 2012; GonzálezSanmamed \& Fuentes-Abeledo, 2011; Hevia, 2010; Zabalza, 2011), siendo necesarias investigaciones centradas, tanto en el maximizar aprovechamiento de las prácticas, como en agrandar el prestigio y la importancia de esta etapa formativa presente en casi la totalidad de las titulaciones universitarias actuales. Tradicionalmente, se han valorado las prácticas externas como un tiempo más relajado y más 
reflexivo que formativo, concepto reduccionista de un periodo de tiempo con infinidad de posibilidades y alicientes formativos.

\section{Agradecimientos}

Planes de Formación e Innovación. Programa de mejora de la Calidad. Proyectos de Innovación de la Universidad de Salamanca: Valoración y análisis de las "prácticas externas" en el grado de Pedagogía. Factores influyentes y seminarios preparatorios. (ID2015/0171)

\section{Referencias}

Alemany, J., \& Perramon, X. (2011). Hacia un Practicum que garantice la calidad: diseño e implementación de un protocolo de seguimiento del Practicum. REDU: Revista de Docencia Universitaria, 9(3), 161.

Bartolome, A. R., Cantón Mayo, I., \& Moral Ferrer, J. M. (2016). Una revisión a los Practicum de Educación desde las tecnologías. Revista Prácticum, 1(1), 40-53.

Boletin Oficial del Estado (2014). RD 592/2014, de 11 de julio, por el que se regulan las prácticas académicas externas de los estudiantes universitarios. BOE num.184

Boletin Oficial del Estado (2007). Ley orgánica 4/2007, de 13 de abril, por la que se modifica la Ley Orgánica 6/2001. BOE num.89

De Miguel, M. (2006). Modalidades de enseñanza centradas en el desarrollo de competencias: orientaciones para promover el Cambio metodológico en el EEES. Oviedo: Universidad de Oviedo.

De Pablos, J. de. (2010). Universidad y sociedad del conocimiento. Las competencias informacionales y digitales. Revista de Universidad y Sociedad de Conocimiento, 7(2), 6-16.

Gavari, E. (2012). Estrategias para la comunicación escrita en el Practicum. Editorial Universitaria Ramon Areces.

González, J. C. (2008). TIC y la transformación de la práctica educativa en el contexto de las sociedades del conocimiento. Revista de Universidad y Sociedad del Conocimiento, RUSC, 5(2), 2-19.

González, X. A., \& Hevia, I. (2011). El Practicum de la Licenciatura de Pedagogía: estudio empírico desde la perspectiva del alumnado. Revista de educación, (354), 209-236. 


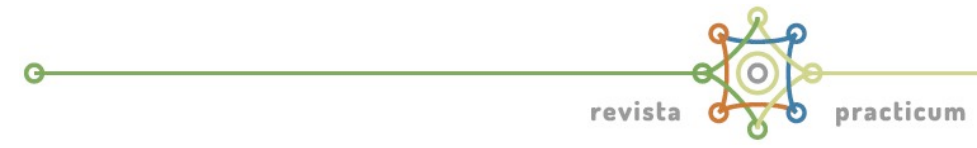

González-Sanmamed, M., \& Fuentes-Abeledo, E. (2011). El Practicum en el aprendizaje de la profesión de docente. Revista de educación, (354), 47-70.

Hernández, J. P. (2014). Actitudes del docente ante la modernización de la Universidad. Un estudio descriptivo correlacional en la Universidad de Salamanca. (Tesis Doctoral). Universidad de Salamanca, Salamanca. Recuperado de https://goo.gl/2ipfQq

Hernández, J. P., Martínez, F., García, F. J., Herrera, E., \& Rodríguez, M.(2013). Teachers' attitude regarding the use of ICT. A factor reliability and validity study. Computers in Humans Behavior, 31, 509-516.

Hevia, I. (2010). Estudio del Practicum de Pedagogía: alternativas metodológicas. Aula abierta, 38(1), 97-108.

Kerlinger, F., \& Lee, H. (2002). Investigación del comportamiento, Métodos de Investigación en Ciencias Sociales (4. ed.). México: McGraw-Hill.

Mendoza, M., \& Covarrubias, C. G. (2014). Valoración del prácticum de los grados del magisterio desde la perspectiva de sus estudiantes. Revista Electrónica Educare, 18(3), 111-142.

Morales, P., Urosa, B., \& Blanco, Á. (2003). Construcción De Escalas De Actitudes «tipo Likert»: Una Guía Práctica. Madrid: Villares de la Reina. La Muralla; Hespérides.

Pérez, R., Galán, A., \& Quintanal, J. (2012). Métodos y diseños de investigación en educación. Editorial UNED.

España. Real Decreto 592/2014, de 11 de julio, por el que se regulan las prácticas académicas externas de los estudiantes universitarios. http://www.boe.es/buscar/doc.php?id=BOE-A-2014-8138

Rodríguez, G., Gil, J., \& García, E. (1996). Metodología de la investigación cualitativa. Archidona: Aljibe.

Valverde, M. A. (2016). Las prácticas externas extracurriculares: tutorización y seguimiento como medida para incrementar su calidad. Practicum, 1(1), 54-79.

Zabalza, M. A. (2011). El Practicum en la formación universitaria: estado de la cuestión. Revista de educación, (354), 21-43.

Zabalza, M. A. (2016). El Practicum y las prácticas externas en la formación universitaria. Revista Prácticum, 1(1), 1-23. 